\title{
Optical data of meteoritic nano-diamonds from far-ultraviolet to far-infrared wavelengths ${ }^{\star}$
}

\author{
H. Mutschke ${ }^{1}$, A. C. Andersen ${ }^{2}$, C. Jäger ${ }^{1}$, T. Henning ${ }^{3}$, and A. Braatz ${ }^{1}$ \\ 1 Astrophysikalisches Institut und Universitäts-Sternwarte (AIU), Schillergäßchen 3, 07745 Jena, Germany \\ e-mail: [mutschke; conny] @astro.uni-jena.de \\ 2 NORDITA, Blegdamsvej 17, 2100 Copenhagen, Denmark \\ e-mail: anja@nordita.dk \\ 3 Max-Planck-Institut für Astronomie, Königstuhl 17, 69117 Heidelberg, Germany \\ e-mail: henning@mpia.de
}

Received 21 October 2003 / Accepted 10 May 2004

\begin{abstract}
We have used different spectroscopic techniques to obtain a consistent quantitative absorption spectrum of a sample of meteoritic nano-diamonds in the wavelength range from the vacuum ultraviolet $(0.12 \mu \mathrm{m})$ to the far infrared $(100 \mu \mathrm{m})$. The nano-diamonds have been isolated by a chemical treatment from the Allende meteorite (Braatz et al. 2000, Meteorit. Planet. Sci., 35, 75). Electron energy loss spectroscopy (EELS) extends the optical measurements to higher energies and allows the derivation of the optical constants $(n \& k)$ by Kramers-Kronig analysis. The results can be used to restrain observations and to improve current models of the environment where the nano-diamonds are expected to have formed. We also show that the amount of nano-diamond which can be present in space is higher than previously estimated by Lewis et al. (1989, Nature, 339, 117).
\end{abstract}

Key words. methods: laboratory - stars: abundances - stars: atmospheres - stars: carbon - ISM: dust, extinction ISM: lines and bands

\section{Introduction}

Presolar meteoritic grains have opened the possibility of studying circumstellar dust grains directly in the laboratory, providing an important complement to astronomical observations. The identification of the presolar origin of the grains is based on their highly anomalous isotopic composition which in general agrees with those expected for stellar condensates (Zinner 1998; Ott 2003). Diamonds were the first presolar grains to be isolated from meteorites (Lewis et al. 1987) and they account for more than $99 \%$ of the identified presolar meteoritic material. Their abundance normalized to the meteorite's matrix is around 500-1000 ppm, depending on the metamorphic grade of the meteorite (Alexander et al. 1990; Huss 1990; Huss \& Lewis 1995). They are identified as presolar grains due to a significant overabundance (compared to the Solar isotopic ratios) of the very heavy $(\mathrm{Xe}-\mathrm{H}){ }^{134} \mathrm{Xe}$ and ${ }^{136} \mathrm{Xe}$ as well as the very light $(\mathrm{Xe}-\mathrm{L}){ }^{124} \mathrm{Xe},{ }^{126} \mathrm{Xe}$ isotopes (together called Xe-HL composition).

The origin of the meteoritic nano-diamonds is still a matter of debate. The Xe-HL anomaly is expected to be produced by $r$ and $p$-process nucleosynthesis in supernovae (SN). Therefore, it has been suggested that the diamonds condense either

* Table 3 is also available in electronic form at the CDS via anonymous ftp to cdsarc.u-strasbg.fr $(130.79 .128 .5)$ or via http://cdsweb.u-strasbg.fr/cgi-bin/qcat?]/A+A/423/983 directly in SN outflows (Clayton 1989) or that they originate from cool red giants and are later implanted with Xe (Jørgensen 1988). As proposed by Jørgensen \& Andersen (1999) it is also possible that the $\mathrm{Xe}-\mathrm{H}$ anomaly can be explained as a small fraction of pure ${ }^{12} \mathrm{C}$ diamonds which originated in SN II. The bulk of the presolar meteoritic diamonds could then have condensed in evolved carbon stars which would account for the bulk ${ }^{12} \mathrm{C} /{ }^{13} \mathrm{C}$ ratio. The content of light isotopes of $\mathrm{Xe}-\mathrm{L}$ is very small (less than 1 atom per $10^{9}$ diamonds), and can be explained as coming from SN I (Lambert 1992) in binary systems where the low mass component is an evolved carbon star (Jørgensen 1988), or as a by-process of the Xe production in SN II (Ott 1996). However, recently Dai et al. (2002) have, based on investigations of interstellar dust particles, suggested that at least some of the meteoritic nano-diamonds were formed in the inner solar nebula and not in a presolar environment. Whether this would be consistent with the oxidizing conditions expected for the solar nebula is an open question.

So far, the only strong observational evidence for the presence of diamonds in other astrophysical environments comes from the identification of the infrared emission bands of hydrogen-saturated diamond surfaces at 3.43 and $3.53 \mu \mathrm{m}$ in certain Herbig-AeBe objects as well as in the post-AGB star HR 4049 (Guillois et al. 1999; van Kerckhoven et al. 2002). The earlier identification by Allamandola et al. (1992), who suggested nano-diamonds as the source of a $3.47 \mu \mathrm{m}$ 
absorption feature seen in dense molecular clouds (protostars), now seems untenable (Brooke et al. 1996, 1999). The $\sim 21 \mu \mathrm{m}$ feature seen in some post-AGB stars (Kwok et al. 1989) is attributed by Hill et al. (1998) to the presence of nano-diamonds, however this feature has also been attributed to several other molecules and solids (for recent assignments see Kwok et al. 1989; Volk et al. 1989; Papoular 2000; von Helden et al. 2000; Speck \& Hofmeister 2003; Posch et al. 2004).

The diamond grains seen in the Herbig-AeBe objects are different from meteoritic ones. The observed emission bands require that the diamonds must be at least one order of magnitude larger than the average size of the meteoritic grains. Nanodiamonds of the size of the meteoritic ones would emit broader bands at about 3.41 and $3.50 \mu \mathrm{m}$ (Sheu et al. 2002; Jones et al. 2004), provided that their surfaces are hydrogenated. Jones et al. (2004) have recently published a new study on the C-H stretching IR bands of surface-processed meteoritic nanodiamonds. They find that the spectra of nano-diamonds of a certain surface structure show similarities with class B emission band sources. They also emphasize that this would strengthen the case for a nano-diamond carrier of the $\sim 21 \mu \mathrm{m}$ emission feature observed in most of the class B objects. Altogether, the current observational and experimental findings, including the presence of the meteoritic nano-diamonds indicate, that diamonds are a constituent at least of certain astrophysical environments. Nano-diamonds with sizes like the meteoritic diamonds could very well be more common than the larger diamonds, due to their detection limitations.

If nano-diamonds are present, they need to be taken into account in models of these environments. Especially, the optical properties (absorption and scattering cross sections) will have to be included in modelling of synthetic spectra. This requires knowledge of the optical constants of the material within a broad wavelength range, i.e. the complex refractive index ( $m=n+\mathrm{i} k$ ) or the dielectric function $\left(\varepsilon=m^{2}\right)$. These data have so far been available only in limited spectral ranges. Lewis et al. (1989) made the attempt to combine measured infrared data with modified literature data of bulk diamonds in the ultraviolet. Part of the UV/Vis and IR spectrum of meteoritic nanodiamonds was measured by Mutschke et al. (1995), Andersen et al. (1998), and Braatz et al. (2000).

With this paper we intend to provide the needed optical data by combining information from optical and EELS measurements on a sample of highly purified meteoritic nano-diamonds extracted from the Allende meteorite (Braatz et al. 2000). After a short summary of the physical and chemical characteristics of the meteoritic nano-diamonds, which are essential to understand their spectroscopic properties, the optical measurements are described in Sect. 3, followed by the EELS measurements and the derivation of the complex refractive index (Sect. 4). In Sect. 5 we discuss the consequences of our new results for the observability of nano-diamonds in astrophysical environments.

\section{Properties of the meteoritic nano-diamonds}

Presolar diamonds are found in relatively unprocessed meteorites, the so-called carbonaceous chondrites, and they account for about $3 \%$ of the total amount of carbon in the meteorite. An in situ search for diamonds in the Allende meteorite (Banhart et al. 1998) showed that they are situated in the matrix of the meteorite, i.e. not related to other inclusions such as the chondrules. A number of impurities have been identified in the meteoritic diamonds, including noble gases ( $\mathrm{He}, \mathrm{Ne}, \mathrm{Ar}, \mathrm{Kr}$, and $\mathrm{Xe}$ ), $\mathrm{Ba}$ and $\mathrm{Sr}$ (which are slightly enriched in $r$-process isotopes; Lewis et al. 1991), H with ${ }^{1} \mathrm{H} /{ }^{2} \mathrm{D}=5193$ (Virag et al. 1989; $\left.\left({ }^{1} \mathrm{H} /{ }^{2} \mathrm{D}\right)_{\text {terrestrial }}=6667\right), \mathrm{N}$ with ${ }^{14} \mathrm{~N} /{ }^{15} \mathrm{~N}=406$ (Russell et al. $\left.1991 ;\left({ }^{14} \mathrm{~N} /{ }^{15} \mathrm{~N}\right)_{\text {terrestrial }}=272\right)$. The ${ }^{12} \mathrm{C} /{ }^{13} \mathrm{C}$ ratio is only slightly higher than the terrestrial one (92 compared to 89; Lewis et al. 1987).

The most intriguing property of the nano-diamonds is their very small size, with a median diameter of less than $20 \AA$ (Fraundorf et al. 1989) and, consequently, the dominance of the grain surface structure in their physical and chemical properties. A typical meteoritic diamond contains on the order of $1000-2000$ carbon atoms, with $\approx 25 \%$ of these belonging to the surface (Jones \& d'Hendecourt 2000). Since the surface atoms have free valences, they can either bind chemical groups or, in the case of carbon atoms, change their hybridization state and restructure the $\mathrm{C}-\mathrm{C}$ bonds, i.e., form unsaturated bonds (for detailed discussions on nano-diamond surface structure see Evans 1992; Jones \& d'Hendecourt 2000, and references therein).

Indications for this restructuring of the surface come from electron energy loss spectroscopy (EELS) as well as from density measurements. Bernatowicz et al. (1990) found that in EELS spectra of nano-diamonds extracted from the Allende and Murray CE meteorites the diamond ( $\sigma$-electron) plasmon was shifted to smaller energies by about $5 \mathrm{eV}$ (see also Sect. 4.1). They explained this behaviour by assuming a grain mantle consisting of hydrogenated amorphous carbon and estimated the volume fraction of this mantle to be 0.46 of the grain volume. Lewis et al. (1987) have measured an effective bulk density of meteoritic nano-diamonds from the Murchison meteorite of $2.22-2.33 \mathrm{~g} \mathrm{~cm}^{-3}$, well below the density of pure diamond (3.51 $\left.\mathrm{g} \mathrm{cm}^{-3}\right)$. Lewis et al. (1989) interpret this as an implication for the presence of residual water and amorphous carbonaceous phases $(\mathrm{a}-\mathrm{C}$ and $\mathrm{a}-\mathrm{C}: \mathrm{H})$, which was taken by Bernatowicz et al. (1990) as support for the core-mantle model, which would give a density of $2.4-2.5 \mathrm{~g} \mathrm{~cm}^{-3}$.

Another clear indication for $\mathrm{sp}^{2}$ carbon is the presence of a $1 \mathrm{~s}-\pi^{\star}$ feature in the carbon core-electron transitions in EELS spectra measured by several authors (Bernatowicz et al. 1990; Blake et al. 1988; Dorschner et al. 1996, see also Fig. 1). The analysis of the strength of the feature in the present sample gives a typical fraction of $\mathrm{sp}^{2} / \mathrm{sp}^{3}$ carbon atoms of 0.11 , i.e. about half of the surface atoms are $\mathrm{sp}^{2}$. This would be consistent with the presence of $\mathrm{C}=\mathrm{C}$ chains at the restructured surface, which has been described by Evans (1994), and with a contribution from $\mathrm{C}=\mathrm{O}$ groups. The abundance of $\mathrm{C}=\mathrm{O}$ groups can be estimated from the IR spectrum $(5.7 \mu \mathrm{m}$ band $)$ to be about $1 / 4$ of the $\mathrm{sp}^{2}$ atoms. The $\mathrm{sp}^{3}$ surface atoms probably largely form $\mathrm{C}-\mathrm{O}-\mathrm{C}$ and $\mathrm{C}-\mathrm{OH}$ groups.

The importance of the surface properties is underlined by infrared spectroscopy results, which reveal a number of bands due to functional groups bound to the nano-diamond surface. These bands have been measured before by several groups on nano-diamonds from different meteorites such as 
Table 1. Measuring methods for spectroscopy of the meteoritic nano-diamonds in different parts of the spectral region.

\begin{tabular}{cccccc}
\hline \hline $\begin{array}{c}\text { Wavelength } \\
(\mu \mathrm{m})\end{array}$ & Preparation & $\begin{array}{c}\text { Column density } \\
\left(\mathrm{mg} / \mathrm{cm}^{2}\right)\end{array}$ & Instrument & $\begin{array}{c}\text { Resolution } \\
\lambda / \Delta \lambda\end{array}$ & Remarks \\
\hline $0.01-0.2$ & On TEM grid & - & EELS (GIF 100) & $200-10$ & - \\
$0.12-0.23$ & On CaF 2 substrate & 0.0169 & VUV (LZ Hannover) & $\sim 500$ & Closed film \\
$0.19-0.5$ & On $\mathrm{CaF}_{2}$ substrate & 0.069 & Lambda 19 (Perkin Elmer) & $\sim 3000$ & Integrating sphere \\
$0.5-2$ & In KBr pellet & 1.81 & Lambda 19 (Perkin Elmer) & $\sim 10000$ & Agglomeration \\
$2-25$ & In KBr pellet & 0.52 & FTIR 113v (Bruker) & $2500-200$ & Agglomeration \\
$25-100$ & In PE pellet & 1.57 & FTIR 113v (Bruker) & $400-100$ & Agglomeration \\
\hline
\end{tabular}

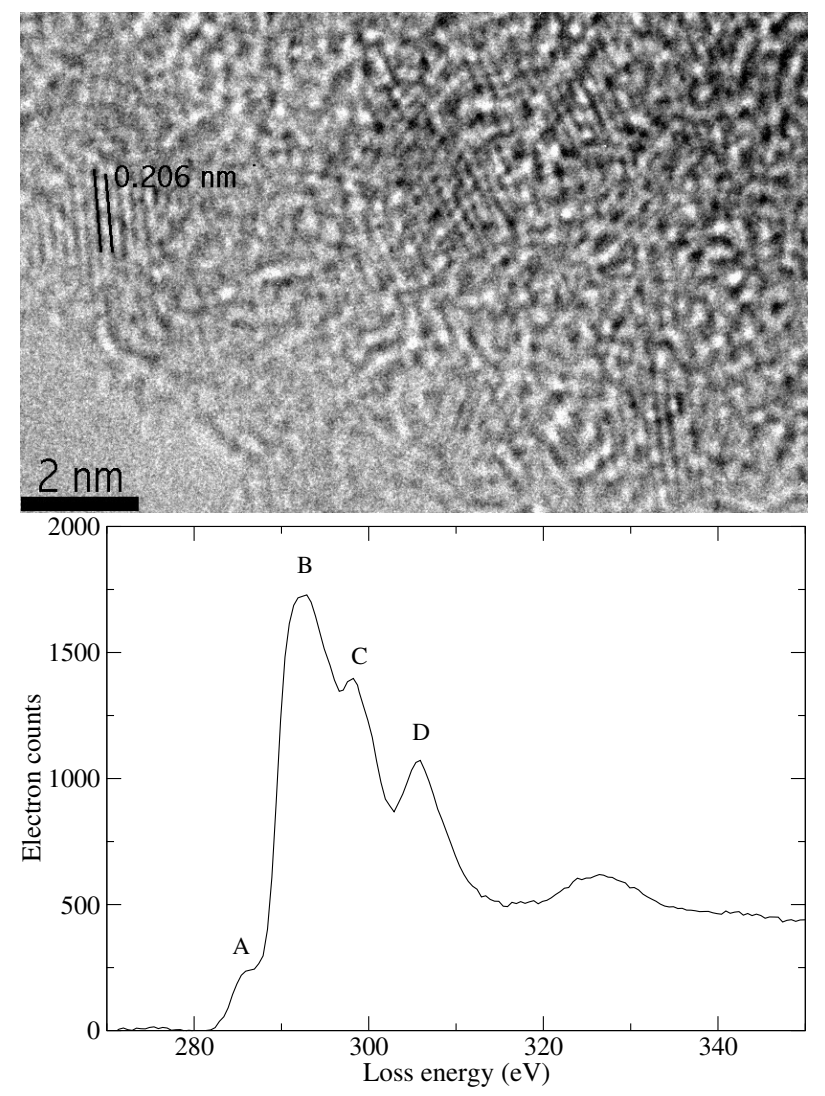

Fig. 1. Upper panel: bright-field transmission electron micrograph showing an agglomerate of meteoritic nano-diamonds from our sample with characteristic fringes corresponding to the (111) spacing of the diamond lattice. Lower panel: EELS core-loss spectrum of the meteoritic nano-diamonds showing the $1 \mathrm{~s}-\sigma^{\star}$ transition bands of diamond (B-D). A denotes the position of contributions from $1 \mathrm{~s}-\pi^{\star}$ transitions.

Allende (Lewis et al. 1989; Koike et al. 1995; Andersen et al. 1998; Braatz et al. 2000), Murchison (Colangeli et al. 1994; Mutschke et al. 1995; Braatz et al. 2000) and Orgueil (Hill et al. 1997). Comparisons of the measurements can be found in Andersen et al. (1998) and Braatz et al. (2000). The assigned bands indicate the presence of oxygen, hydrogen, and nitrogen in different configurations at the diamond surface.

As mentioned before, the results of the isotopic analysis (Russell et al. 1991; Virag et al. 1989) imply that at least some of the $\mathrm{H}$ and $\mathrm{N}$ must be presolar. However, by
IR spectroscopy it has not yet been possible to identify surface groups which originate from the astrophysical environment in which the grains have either been formed or processed. Before it is possible to measure the spectral properties of meteoritic diamonds, it is necessary to extract them from the rest of the meteoritic material. This means that $99.9 \%$ of the meteorite has to be removed in a destructive chemical separation, in which undesirable minerals are dissolved by appropriate reagents. Hill et al. (1997) and Braatz et al. (2000) showed that e.g. the oxidizing steps of the extraction procedure should be able to modify the surface structure.

The chemical separation procedure we have used, is a variant of those developed by Tang \& Anders (1988) and Amari et al. (1994) and is described in Braatz et al. (2000) together with infrared spectra taken after each step for a sample of Murchison diamonds. The nano-diamond sample used for the measurements presented here is identical to the Allende sample of Braatz et al. (2000). The diamonds were extracted from $114 \mathrm{~g}$ of the Allende meteorite. The extraction procedure is the same for these. The IR spectra are reported in Braatz (2000). The yield of the final diamond sample was $16 \mathrm{mg}$.

\section{Optical spectroscopy}

We have performed optical transmission measurements of meteoritic nano-diamonds in the wavelength range $0.12-100 \mu \mathrm{m}$, which required the use of different spectrometers and preparation methods. To prepare suitable samples, the diamonds had to be either dispersed in or deposited on media which are transparent in the corresponding part of the spectral range. Because of the strong variation in the mass absorption coefficient from the vacuum-ultraviolet to the far-infrared wavelengths, samples with different column densities of nano-diamonds had to be prepared. The intended quantitative absorption measurements require as exact a knowledge of the column densities as possible.

Dispersion in liquids failed, because the diamonds exhibited a strong tendency to aggregate and to sediment, regardless of the $\mathrm{pH}$-value or kind of liquid. We therefore used the following methods: (1) the sample was dispersed in $\mathrm{KBr}$ and polyethylene pellets for measurements in the visible and the infrared spectral ranges and (2) the sample was deposited onto $\mathrm{CaF}_{2}$ windows from a suspension for measurements in the UV. Table 1 summarizes the methods and instruments applied for each of the different spectral regions. 


\subsection{Measurements on films on $\mathrm{CaF}_{2}$ substrate}

For the vacuum-ultraviolet (VUV) and the ultraviolet/visible (UV/Vis) measurements, four diamond-film samples were produced by transferring $3.7,8.3,34.4$, and $63.3 \mu \mathrm{g}$, respectively, of nano-diamonds from an aqueous colloidal $(\mathrm{pH}=6.8)$ to the surface of $\mathrm{CaF}_{2}$ windows. After drying, the nano-diamonds formed closed films of 7-8 mm diameter. The covered area was measured under an optical microscope. The deposited mass was determined with an accuracy of $\pm 0.05 \mu \mathrm{g}$ by weighing of the windows before and after deposition of the diamonds. The thickness homogeneity of the films was checked by optical transmission measurements at different positions and was found to be better than $\pm 20 \%$.

In the UV and visible (190-800 nm), a Perkin Elmer Lambda 19 spectrometer was used for measuring the transmission through the diamond films. The VUV spectrometer was a double-beam grating spectrometer built by Laserzentrum Hannover, using a deuterium lamp with $\mathrm{MgF}_{2}$ window as the light source. The wavelength range of this spectrometer is $115-230 \mathrm{~nm}$ (accuracy $0.2 \mathrm{~nm}$ ), but the range of the measurements was limited by the $\mathrm{CaF}_{2}$ substrates to $\geq 124 \mathrm{~nm}$. The VUV and UV/Vis transmission spectra measured on the same sample always agreed within a relative error of $\pm 5 \%$ in the overlap region.

In order to eliminate substrate influences, in the VUV the transmission was measured at each of the $\mathrm{CaF}_{2}$ windows before and after sample deposition. The ratio of these spectra is the transmittance of the diamond film, which contains information about the reflection, the scattering and the absorption by the film. With the Lambda 19 spectrometer, we are able to disentangle these different loss mechanisms by additional measurements using an integrating sphere detector (spectral range $0.25-2.0 \mu \mathrm{m})$. For these measurements, the sample is placed inside the integrating sphere and the intensity coming from the illuminated sample is collected and compared to the intensity of a reference beam. Opening/closing of ports for the transmitted and specularly reflected beams makes it possible to measure the scattered light individually or together with these contributions. Closing of all ports allows a direct measurement of the intensity absorbed by the sample ("absorption mode").

The measurements using the integrating sphere revealed a reflectance of the samples of the order of $13 \%$ at $\lambda=800 \mathrm{~nm}$, which is expected for a film with a refractive index of about 2 on $\mathrm{CaF}_{2}$ substrate (see Fig. 2). This value decreased with decreasing wavelength, accompanied by an increase in the intensity of the diffusely scattered light. For the thinner films, scattering plus reflection increased only slightly toward smaller wavelengths, whereas for thicker films it increased up to $22 \%$ at $\lambda=300 \mathrm{~nm}$. We interpret this as a higher surface roughness of the thicker films.

The correction of the transmission spectra for the scattering and reflection effects was done by taking the reduction of the incident intensity by reflection and scattering losses into account, i.e. by dividing the original spectra by $1-\mathrm{R}-\mathrm{S}$, R being the reflectance and $\mathrm{S}$ the scattering. For this purpose, the $\mathrm{R}+\mathrm{S}$ spectra have been fitted either by a linear or a $\lambda^{-1 / 2}$ function and extrapolated to the VUV wavelengths. The resulting

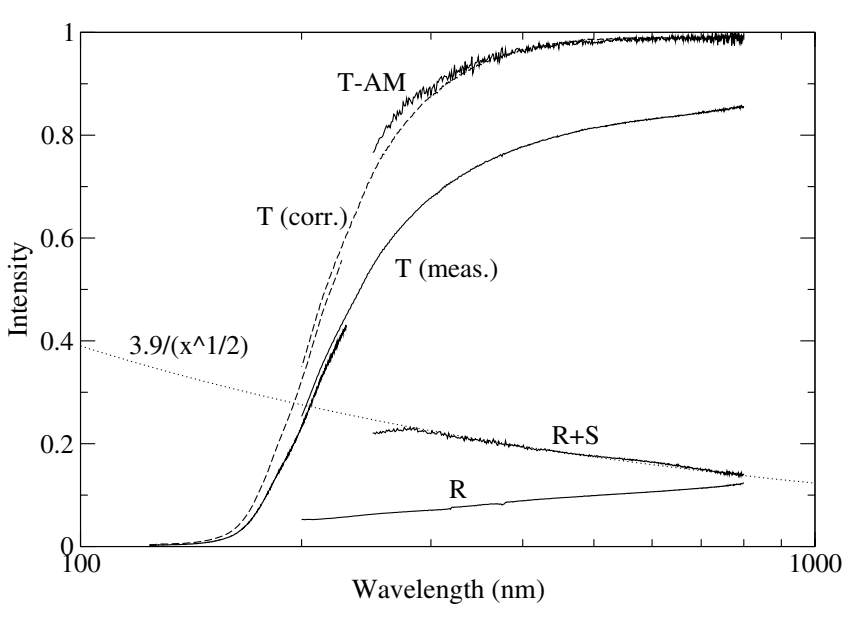

Fig. 2. Illustration of the scattering correction for the VUV and $\mathrm{UV} / \mathrm{Vis}$ transmission spectra $\mathrm{T}$ (meas.) measured at the $69 \mu \mathrm{g} \mathrm{cm}^{-2}$ diamond film. The solid lines represent the original measurements, where the "reflectance plus scattering" $(\mathrm{R}+\mathrm{S})$, and "absorption mode" (T-AM) spectra have been measured using an integrating sphere. The dotted line is a fit to the $\mathrm{R}+\mathrm{S}$ spectrum, which has been used to produce the corrected spectra (T/(1-R-S), dashed lines). The corrected UV/Vis spectrum corresponds well to the "absorption mode" integrating sphere measurement.

corrected spectra agree well with the spectra measured in the "absorption mode" (see Fig. 2). However, because of the narrower spectral region and the stronger noise, the latter have not been used.

For the final spectrum, in the VUV (wavelength range $0.12-0.23 \mu \mathrm{m}$ ), the spectrum measured at the $8.3 \mu \mathrm{g}$ sample (column density $\sigma=16.9 \mu \mathrm{g} \mathrm{cm}^{-2}$ ) was selected, because this was the thickest film being still transparent at the shortest wavelengths. For the UV/Vis wavelengths up to $0.5 \mu \mathrm{m}$, the spectrum of the $34.4 \mu \mathrm{g}$ sample (column density $\sigma=69 \mu \mathrm{g} \mathrm{cm}{ }^{-2}$ ) was used. For significant measurements at larger wavelengths, the absorption by these films was too low. The thickest film $(63.3 \mu \mathrm{g})$ exhibited a tendency to flake and was not used.

The transmission $T$ was converted to the mass absorption coefficient $\kappa_{\text {film }} \equiv \alpha / \rho=\ln (1 / T) / \sigma$. Here, $\rho$ is the mass density and $\alpha$ is the volume absorption coefficient which for a continuous material is given by $\alpha=4 \pi k / \lambda$ with $\lambda$ being the wavelength and $k$ being the imaginary part of the complex refractive index.

\subsection{Measurements on $\mathrm{KBr}$ and polyethylene pellets}

The spectrum in the visible and near-infrared range up to a wavelength of $2 \mu \mathrm{m}$ were measured again with the Lambda 19 spectrometer equipped with the integrating sphere. The sample here consisted of $2.4 \mathrm{mg}$ of nano-diamonds, mixed thoroughly with powdered $\mathrm{KBr}$ and pressed into a pellet of $13 \mathrm{~mm}$ diameter. The column density of the nano-diamonds was thus $\sigma=1.81 \mathrm{mg} \mathrm{cm}^{-2}$. The pellet showed very strong diffuse scattering of up to $50 \%$ of the incident intensity, probably caused by big diamond aggregates concentrated around the $\mathrm{KBr}$ grains and by the high refractive index of the nanodiamonds compared to the $\mathrm{KBr}$ matrix. Consequently, apart 
from measuring the scattered intensity $S$, the "absorption mode" (see Sect. 3.1) of the integrating sphere was used to determine the absorbed intensity $A$. Since the scattering here is a volume effect in contrast to the scattering from the diamond film, scattering and absorption have to be treated as directly competing mechanisms, and the mass absorption coefficient can be calculated from

$\kappa_{\mathrm{abs}}=-\frac{1}{\sigma} \ln (1-A-S) \times \frac{A}{A+S}$.

The calculated mass absorption coefficient is used in the final spectrum in the wavelength range $0.5-2 \mu \mathrm{m}$. At shorter wavelengths, it starts to fall below the values expected from the film measurements because of saturation effects in the big clusters.

The measurements with the integrating sphere demonstrate that at infrared wavelengths nearly all the continuum extinction produced by nano-diamonds in $\mathrm{KBr}$ pellets is due to light scattering. At a wavelength of $2 \mu \mathrm{m}, \kappa_{\mathrm{abs}}$ has fallen to about $20 \mathrm{~cm}^{2} \mathrm{~g}^{-1}$, whereas the extinction measured with the pellets is typically many $100 \mathrm{~cm}^{2} \mathrm{~g}^{-1}$, even for pellets with a lower diamond concentration (see Braatz et al. 2000). This was also noticed by Andersen et al. (1998), but a quantitative determination of the continuum absorption coefficient requires the integrating sphere technique and was not possible at that time.

Since the use of the integrating sphere is limited to wavelengths below $2 \mu \mathrm{m}$, at larger wavelengths quantitative continuum absorption measurements with the $\mathrm{KBr}$ pellet technique have not been possible. However, with other techniques we did not succeed in producing the diamond column density of $\sim 10 \mathrm{mg} \mathrm{cm}^{-2}$ required for continuum measurements at mid-IR wavelengths. Therefore, for extending the diamond spectrum to the mid-IR, we have corrected the available spectra from Braatz (2000) for the scattering background and fitted them to the near-IR spectrum at $\lambda=2 \mu \mathrm{m}$ assuming that the slope of the spectrum between 1 and $2 \mu \mathrm{m}$, which is approximately proportional to $\lambda^{-1}$, would continue throughout the infrared. This slope is justified for nano-grains with disordered surfaces (Seki \& Yamamoto 1980), and agrees also well with the results of simple model calculations taking the disordered surface into account (see Fig. 7).

\subsection{Description of the final spectrum}

The combined optical absorption spectrum is characterized by a smooth curve throughout the UV, visible and near IR. In the UV at a wavelength of $200 \mathrm{~nm}$, the mass absorption coefficient reaches about $10^{4} \mathrm{~cm}^{2} \mathrm{~g}^{-1}$, which is at least one order of magnitude less than for amorphous carbon but higher than for terrestrial diamonds. It is also higher by about a factor of two than the values reported by previous studies (Mutschke et al. 1995; Andersen et al. 1998; Braatz et al. 2000) for nanodiamonds dispersed in liquids. An explanation for this difference could be the sedimentation of diamond aggregates from the liquid lowering the actual concentration and therefore the measured absorption. Another explanation could be the influence of the thin-film geometry. This will be discussed further below.
Table 2. Assigned absorption bands in the infrared spectra of the diamonds from the Allende meteorite.

\begin{tabular}{ccl}
\hline \hline $\begin{array}{c}\lambda \\
(\mu \mathrm{m})\end{array}$ & $\begin{array}{c}1 / \lambda \\
\left(\mathrm{cm}^{-1}\right)\end{array}$ & Vibrational groups \\
\hline 2.95 & 3400 & OH stretching \\
5.70 & 1760 & $\mathrm{C}=\mathrm{O}$ stretching (Ester) \\
6.2 & 1610 & OH deformation, \\
& & $\mathrm{C}=\mathrm{C}$ stretching \\
$7-11$ & $1400-900$ & C-O-C (Ether) \\
& & C-O $(\mathrm{H})$ \\
& & $\mathrm{C}=\mathrm{C}$ deformation \\
& & C-C stretching \\
19.7 & 508 & Chromite \\
\hline
\end{tabular}

There is no evidence for any of the features in the 200-300 nm range, which were reported by Mutschke et al. (1995), Andersen et al. (1998) and Braatz et al. (2000) with meteoritic diamonds. Whereas the feature at about $220 \mathrm{~nm}$ has only been seen by Mutschke et al. (1995) and is probably due to a contamination, the $270 \mathrm{~nm}$ feature was detected by all authors and was ascribed by Braatz et al. (2000) to singly distributed N atoms. However, Andersen et al. (1998) noted that the feature was much weaker in Allende than in Murchison nano-diamonds, which is consistent with the lower $\mathrm{N}$ concentration (Russell et al. 1991). In previous measurements on the same sample as studied here, but dispersed in liquid, one of us found a very weak indication of enhanced absorption around $300 \mathrm{~nm}$ (Braatz 2000).

It is possible that this weak band is also present in our result but that it is too weak to be detected on the background of the absorption edge. The difference in the spectra published by Andersen et al. (1998), and Braatz et al. (2000) and this study, however, leaves the possibility open that this band might be due to contamination as well. Graphitic carbon, e.g., would be able to cause a band at a similar position. For the purpose of this paper, this question is not very important because the influence of the band on the broad-band optical properties is only marginal.

In the IR, there appear absorption bands due to excitation of surface groups. The positions of the bands as well as their assignment are summarized in Table 2 . They are clearly dominated by oxygen in carboxyl, carbonyl, and C-O-C groups. The influence of the isolation procedure on these bands is discussed in detail by Mutschke et al. (1995), Hill et al. 1997 and Braatz et al. (2000). This will not be repeated here.

It is an open question as to which of the features could have originated from the presolar history of the diamonds. There is an ongoing discussion on the possible formation sites of nano-diamonds; carbon-rich AGB stars (Jørgensen \& Andersen 1999), supernovae (Clayton 1989; Ott 1996), the ISM (Tielens et al. 1987) and the Solar nebula (Dai et al. 2002) have been suggested. The surface features of the nano-diamonds should first reflect the chemical environment at their place of formation. In carbon-rich AGB stars the dangling bonds of the diamond surface should be expected to primarily be chemically saturated by hydrogen and deuterium. In contrast to that, the other formation places are oxygen-rich which will lead to the 


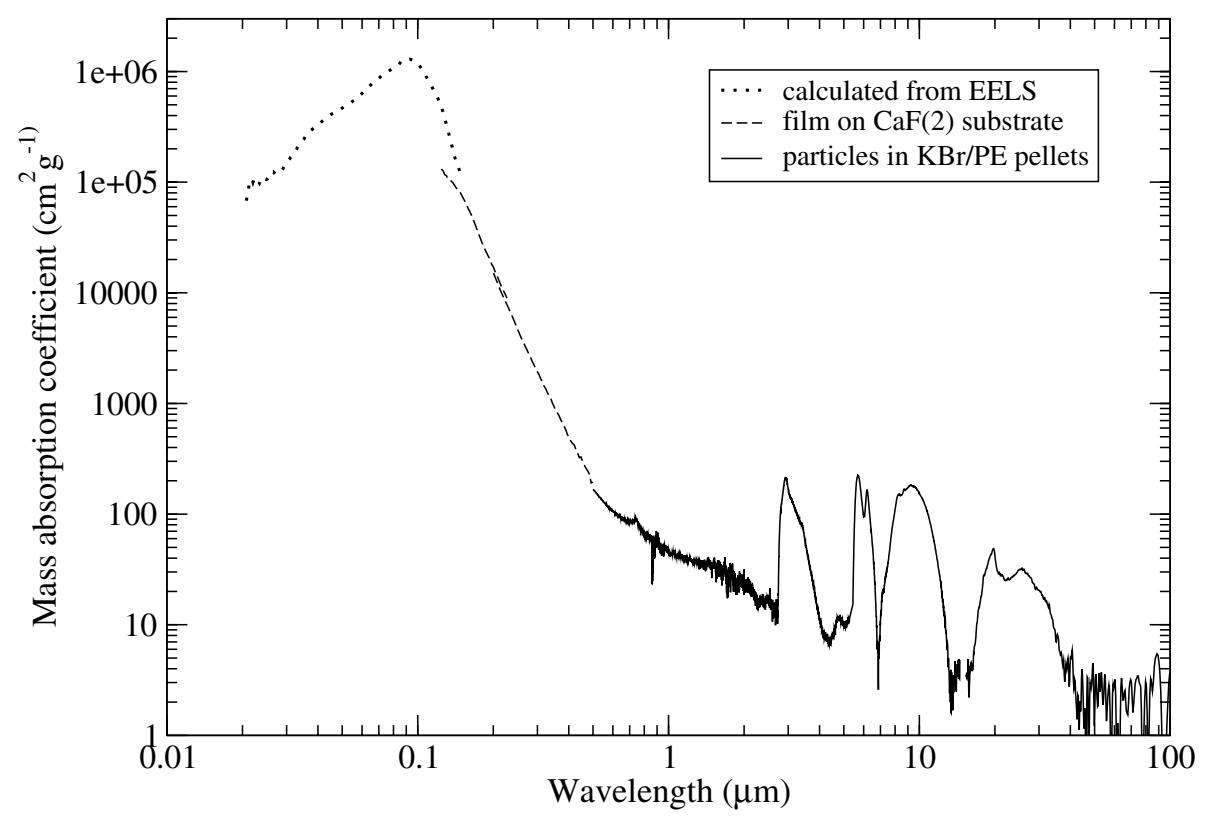

Fig. 3. The combined optical spectra are characterized by a smooth curve throughout the UV, visible, and near infrared. In the IR absorption bands appear due to the excitation of surface groups (see Table 2 for the identification). The "artificial" far-UV spectrum, calculated from the EELS data, shows the inter-band electronic transitions produced by the carbon $\sigma$ electrons. At larger wavelengths, there is no evidence for $\pi$ electronic transitions or bands caused by nitrogen defect centers.

formation of oxygen-containing functional groups at the surface as well as the graphitization of the surface due to catalytic reactions (Evans 1992).

Furthermore, the surface structure is likely to be changed by interaction with UV photons, atoms, radicals, ice mantles, and so on, when exposed to various environments in the ISM. Nano-diamonds are therefore likely to show different spectral features depending on their astrophysical environment. It is also possible that the surface was changed during the incorporation in the meteorite, during solar system formation. However, it was shown by Virag et al. (1989) that the presolar diamonds also carry anomalous hydrogen with ${ }^{1} \mathrm{H} /{ }^{2} \mathrm{D}=5193$ (terrestrial $=6667$ ) implying that at least some of the surface groups are presolar.

The presented IR spectrum as well as most published IR data of meteoritic nano-diamonds represents well oxidized nano-diamonds. The potential user of the data needs to critically judge if these IR bands or possibly bands originating from another kind of surface species are likely to be produced by nano-diamonds in the environment under investigation.

\section{Derivation of optical constants}

\subsection{EELS measurement}

Electron energy loss spectroscopy (EELS) has been used before by Bernatowicz et al. (1990) for the characterization of meteoritic nano-diamonds. It is performed by a magnetic prism spectrometer positioned beneath the viewing screen of a transmission electron microscope (TEM). During penetration of a thin sample, part of the electrons of the primary beam lose an energy $\Delta E$ due to inelastic scattering at valence or core electrons of the sample material. The probability for such processes is proportional to the so-called loss function $\mathfrak{J}(-1 / \varepsilon(\Delta E, \hbar q))$, where $\varepsilon(\Delta E, \hbar q)$ is the complex dielectric function of the material and $\mathfrak{J}$ denotes the imaginary part. The quantity $\hbar q$ is the momentum transfer to the materials' electron system in the scattering process. Resonances of the loss function occur for energies where the real part of the dielectric function vanishes $(\mathfrak{R}(\varepsilon)=0)$ and collective excitations of the valence electrons (plasmons) are possible. This "plasmon loss" as well as losses due to interband transitions occur at loss energies lower than $100 \mathrm{eV}$, whereas at higher energies core electrons can be excited to states above the Fermi level ("core loss").

$\varepsilon(\Delta E, \hbar q)$ corresponds to the optical dielectric function $\varepsilon=m^{2}$ ( $m$ being the complex refractive index) at wavelength $\lambda=\mathrm{hc} / \Delta E$ under the condition that $q \approx 0$, which restricts the scattering angle to small values. For practical purposes, $q \leq 0.1 \AA^{-1}$ is sufficient (Pflüger et al. 1991), at larger angles, deviations in the positions and widths of the plasmon peaks have been measured for carbonaceous materials (Falke et al. 1995). This technique, thus, allows to derive the optical data of a material in the energy range of the strong electronic transitions up to $100 \mathrm{eV}$, corresponding to wavelengths down to $0.01 \mu \mathrm{m}$.

We have prepared the Allende nano-diamonds for EELS measurements by dispersing them in ethanol and transferring them onto a TEM copper grid covered with a porous ("lacey") carbon film. Measurements have been done exclusively on particle aggregates located over film holes. The EELS spectra were obtained with a Philips CM200FEG highresolution TEM operated in the diffraction mode (camera length 400-4700 mm), using a Gatan Imaging Filter (GIF 100) with $0.6 \mathrm{~mm}$ entrance aperture. Primary electron energies were $200 \mathrm{keV}$ and the energy resolution was $0.9 \mathrm{eV}$ based on the fullwidth-half-maximum (FWHM) of the zero energy loss peak. 
The acceptance angle for scattered electrons in the measurements was 0.4 mrad corresponding to a maximum momentum transfer of $q \approx 0.1 \AA^{-1}$. The spectra were corrected for the influence of the energy distribution of the primary beam as well as for plural scattering events following the procedures described in Egerton (1996). The contribution of the primary and elastically scattered electrons ("zero loss") was removed from the spectrum by assuming a symmetric energy distribution of these electrons.

A typical corrected energy loss spectrum of the meteoritic nano-diamonds is shown in Fig. 4. It is similar to the ones obtained by Bernatowicz et al. (1990) but the plasmon peak is found at a smaller energy loss of $26.5 \mathrm{eV}$ compared to about $29 \mathrm{eV}$, which is a consequence of the restriction of the momentum transfer. Compared to bulk diamond (peak at $33.5 \mathrm{eV}$ ), the feature is strongly shifted and broadened. According to Bernatowicz et al. (1990), this can be understood as due to surface carbon atoms deviating in their bonding type from the volume atoms and a mixing of the corresponding dielectric functions. At the signal-to-noise level achievable with these angle-resolved EELS measurements, it is not possible to detect or exclude features due to $\pi$-electron transitions, which would occur at energies below $8 \mathrm{eV}$. In measurements performed with a much larger acceptance angle (in imaging mode), however, distinct $\pi$-electron features are typically not observed with our sample. For the determination of the dielectric function, the loss function at energy losses lower than $8 \mathrm{eV}$ has not been used.

From the loss function $\operatorname{Im}(-1 / \varepsilon(\Delta E, \hbar q \approx 0))$, the dielectric function $\varepsilon(\lambda)$ can be determined via Kramers-Kronig analysis, if the spectrum can be normalized in a proper way. The possibility for the normalization is e.g. provided by assuming $\mathfrak{R}(\varepsilon)$ at low energies, the inverse value of which is related to the integral over the loss function weighted with $\lambda$ (Pflüger et al. 1991). The value of $\mathfrak{R}(\varepsilon)$ at low energies is identical to the square of the refractive index and can be determined from optical measurements in the visible. We adopted for $\mathfrak{R}(\varepsilon)$ a value of 4.0, estimated by Lewis et al. (1989) from density measurements for the meteoritic nano-diamonds. From the normalized loss function, $\varepsilon(\lambda)$ has been calculated in the wavelength range 10-200 nm. Further, this preliminary dataset has been converted into a synthetic absorption spectrum of nano-diamonds in an environment with $\varepsilon_{\mathrm{m}}=2.3$, which completes the optical absorption spectrum as shown in Fig. 3.

\subsection{Optical constants}

Before the combined absorption spectrum, shown in Fig. 3, can be used to derive a consistent wavelength-dependent dielectric function, we have to ask whether the thin-film and small-particle absorption spectra are compatible or can be transformed into each other. Although they match quite well in our measurements, the quantities $\kappa_{\text {film }}$ and $\kappa_{\text {particle }}$ (for spherical grains which are small compared to the wavelength) are

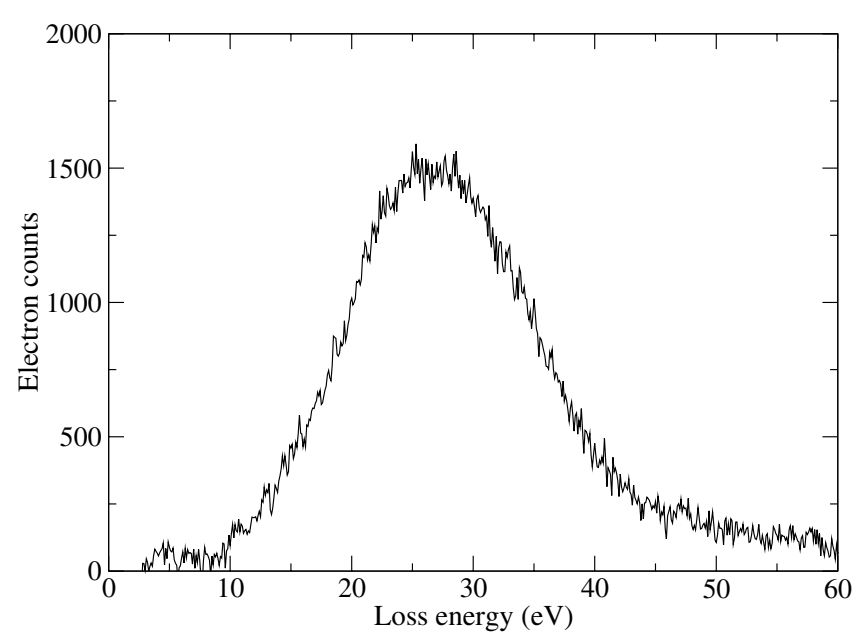

Fig. 4. A typical EELS spectrum of the Allende nano-diamonds. The maximum of the $\sigma$ plasmon $(26.5 \mathrm{eV})$ is displaced compared to bulk diamonds $(33.5 \mathrm{eV})$. This is due to a contribution from carbon atoms of a lower state of hybridization resulting in a lower electron density.

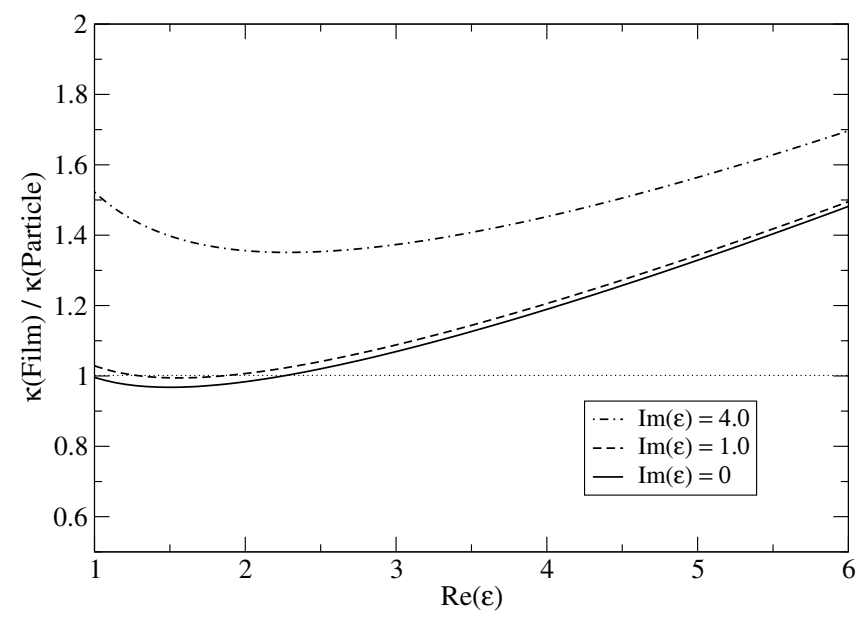

Fig. 5. Simulated ratio of thin-film and small-particle absorption depending on the dielectric function of the material.

related to the dielectric function $\varepsilon$ of the material by different expressions:

$\kappa_{\text {film }} \rho=\frac{4 \pi k}{\lambda}=\frac{4 \pi}{\sqrt{2} \lambda} \sqrt{|\varepsilon|-\mathfrak{R}(\varepsilon)}$.

$\kappa_{\text {particle }} \rho=\frac{6 \pi \sqrt{\varepsilon_{\mathrm{m}}}}{\lambda} \mathfrak{J} \frac{\varepsilon-\varepsilon_{\mathrm{m}}}{\varepsilon+2 \varepsilon_{\mathrm{m}}}$

with $\varepsilon_{\mathrm{m}}$ describing the surrounding medium (Bohren \& Huffman 1983). The ratio of $\kappa_{\text {film }}$ and $\kappa_{\text {particle }}$ for a range of complex $\varepsilon$-values and for $\varepsilon_{\mathrm{m}}=2.3$, which is approximately valid for both $\mathrm{KBr}$ and polyethylene matrices, is shown in Fig. 5. It demonstrates that the deviation between both quantities for nano-diamonds with an assumed $\mathfrak{R}(\varepsilon)$ of 4.0 is of the order of $20 \%$ in spectral ranges where the absorption of the material is low $(\mathfrak{J}(\varepsilon)<1.0)$. In the band edge, where both $\mathfrak{R}(\varepsilon)$ and $\mathfrak{J}(\varepsilon)$ increase, the deviation can reach $50 \%$ or more, whereas at even shorter wavelengths, where anomalous dispersion occurs and $\mathfrak{R}(\varepsilon)$ falls down to values of 1.0 

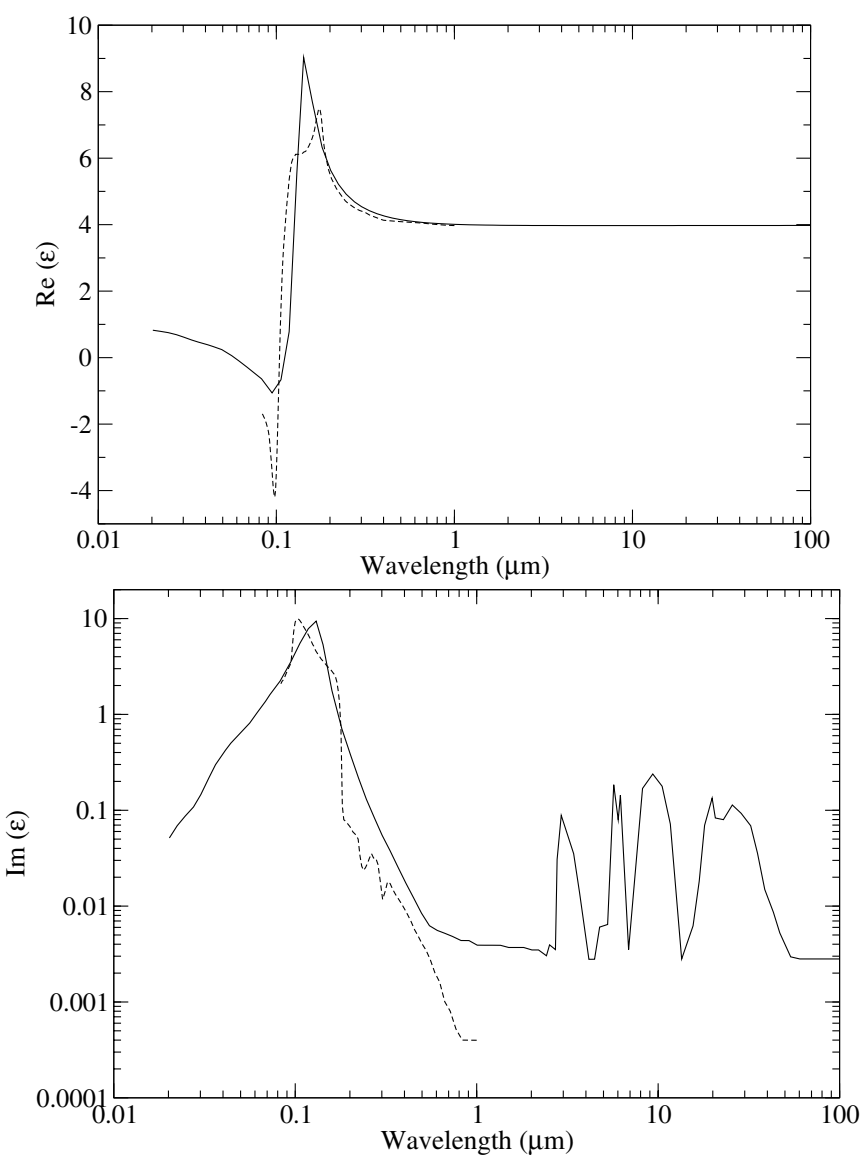

Fig. 6. Dielectric function (upper panel: real part, lower panel: imaginary part) of the meteoritic nano-diamonds (solid line) compared to the previous diamond data (dashed line) published by Lewis et al. (1989).

and smaller, the deviation is reduced again. This means that at wavelengths smaller than about $150 \mathrm{~nm}$ the steepness of the thin-film spectrum should decrease, what indeed seems to be observed (see Fig. 3).

We have accounted for these effects by dividing the thinfilm spectra by a factor of 1.3 and by not using them below $\lambda=0.18 \mu \mathrm{m}$. After that, the different parts of the spectrum including the synthetic far-UV spectrum have been merged into one (using linear interpolation if necessary) at a reduced resolution, with equidistant points on the logarithmic scale. From this spectrum, the dielectric function has been deduced by using the Kramers-Kronig transformation based on Eq. (3) developed by Ossenkopf et al. (1992).

The resulting dataset is shown in Fig. 6 compared to the previous data by Lewis et al. (1989). In the UV and visible, the Lewis et al. data deviate from our new results. They are based on modified bulk data rather than on measurements and do not take into account the nano-particle character of the meteoritic diamonds. In astronomical modelling, usually the complex refractive index $m=n+\mathrm{i} k=\sqrt{\varepsilon}$ is used instead of the dielectric function to describe the optical material properties. Therefore, we give these values in Table 3 .

\section{Discussion}

Observations of nano-diamonds in space would be very desirable first for the clarification of their origin and evolution, and second for the determination of their abundance. Unfortunately, with the exception of the diamond detection in a few Herbig $\mathrm{Ae} / \mathrm{Be}$ objects and one post-AGB star (Guillois et al. 1999), there were no positive results yet. There might be a number of reasons for this; one is that the intrinsic features longward of the electronic band edge are very weak, even for nitrogendoped diamonds. A second reason is that surface groups may produce strong bands, but that it may be hard to distinguish them from bands produced by other carbonaceous matter. Moreover, the actual surface structure of nano-diamonds in space is unknown and is likely to depend on the environment. Finally, the diamonds may be covered or incorporated into larger grains, which would alter their signature or prevent it from becoming visible.

One chance for the detection is the tiny size of the nanodiamonds, which opens the possibility of stochastic heating to temporarily high temperatures by single UV-photon absorption. Consequently, they could become visible in emission, if the nano-diamonds would be separated from other grains. In the case of the diamond emission bands seen in the Herbig $\mathrm{Ae} / \mathrm{Be}$ objects, it has been possible to derive an abundance of the diamonds which, however, is very low (1 part per billion relative to hydrogen, van Kerckhoven et al. 2002). This abundance value, however, may reflect only a population of diamond grains in a narrow size range which allows both the stochastic heating process and the presence of well-ordered surfaces. The abundance of nano-diamonds in primitive meteorites is, as we know, much higher (500-1000 ppm), but one must bear in mind the possibility that the concentration of interstellar diamonds in meteorites could be disproportionately high as a result of their superior chemical and physical durability (Sandford 1996).

A more exact way to derive upper limits of the diamond abundance in space is provided by the ultraviolet absorption of the diamonds. Lewis et al. (1989) suggested that it would be compatible with the measured interstellar extinction (e.g. Stecher 1965; Fitzpatrick \& Massa 1986; Clayton et al. 2003 ), if $<10 \%$ of the carbon would be present as diamonds. Interestingly, the comparison of mass absorption coefficient spectra in Fig. 7 reveals that the Lewis et al. data are erroneous because they produce higher absorption values than the original bulk diamond data, contrary to what had been intended. The reason for this is that Lewis et al. (1989) reduced the imaginary part of the dielectric function to account for the reduced density of the meteoritic diamonds instead of reducing the polarizability. Our results give a value of the ultraviolet extinction of meteoritic nano-diamonds at the Lyman limit which is about a factor of 1.5 less than the estimate by Lewis et al. (1989). Thus, the restrictions on the amounts of diamonds which can be present in space are less strict than previously suggested. Even larger masses of big diamonds could be hidden in the ISM, in principle.

Another constraint on the abundance of nano-diamonds comes from the lack of structure in the observed galactic 


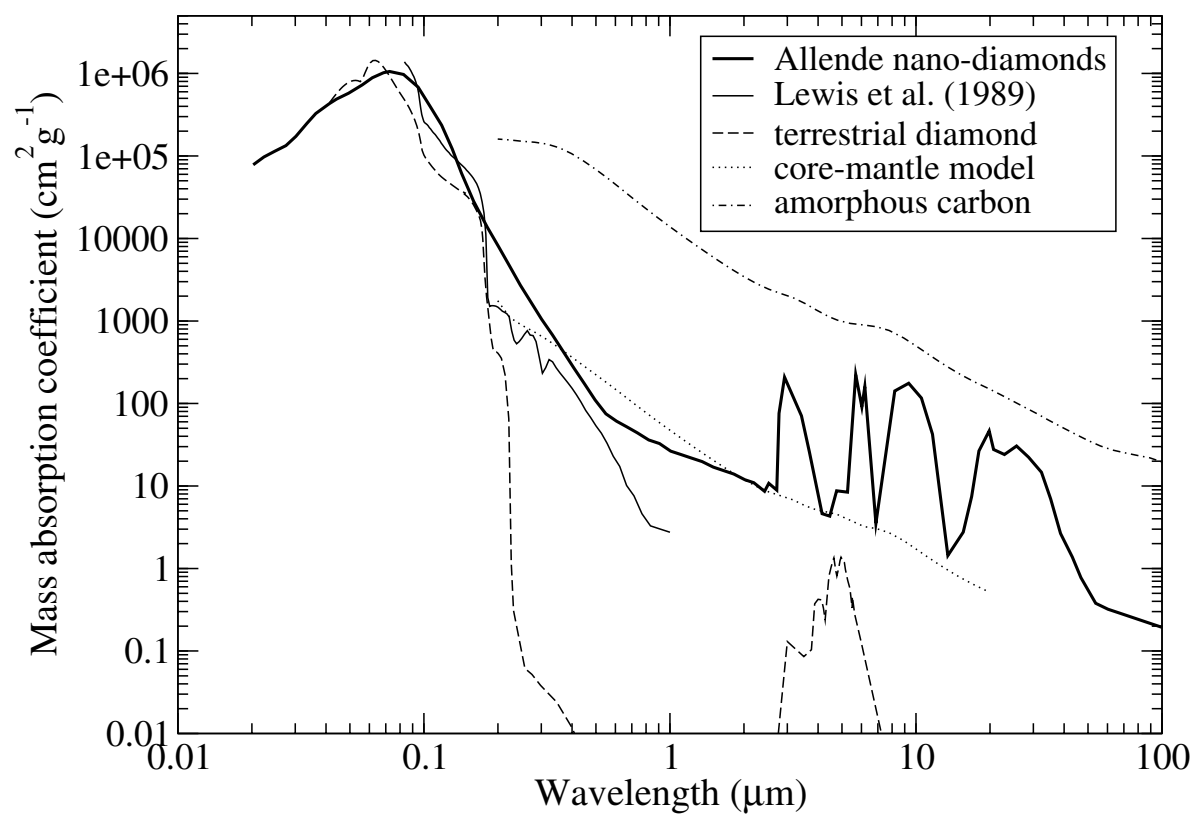

Fig. 7. Mass absorption coefficient spectra of the meteoritic nano-diamonds calculated from our data and from the data of Lewis et al. (1989). For comparison, the simulated absorption spectra of terrestrial diamond particles (data from Edwards \& Philipp 1985), amorphous carbon particles (data "cel400" from Jäger et al. 1998) and of a core-mantle particle with terrestrial diamond core (99\% volume fraction) and amorphous carbon mantle (1\% volume fraction) are shown. Using a more graphitic mantle material such as "cel1000" (not shown), results in a somewhat flatter IR continuum slope.

Table 3. The optical constants $n$ and $k$ of meteoritic nano-diamond, $\lambda$ is the wavelength, $Q / a$ is the extinction efficiency.

\begin{tabular}{|c|c|c|c|c|c|c|c|c|c|c|c|}
\hline $\begin{array}{c}\lambda \\
\mu \mathrm{m}\end{array}$ & $\begin{array}{c}Q / a \\
\mathrm{~cm}^{-1}\end{array}$ & $n$ & $k$ & $\begin{array}{c}\lambda \\
\mu \mathrm{m}\end{array}$ & $\begin{array}{c}Q / a \\
\mathrm{~cm}^{-1}\end{array}$ & $n$ & $\bar{k}$ & $\begin{array}{c}\lambda \\
\mu \mathrm{m}\end{array}$ & $\begin{array}{c}Q / a \\
\mathrm{~cm}^{-1}\end{array}$ & $n$ & $k$ \\
\hline 0.0202 & 2.399E5 & 0.908 & 0.0283 & 0.4493 & 531.6 & 2.049 & 0.0030 & 6.1957 & 490.4 & 1.993 & 0.0361 \\
\hline 0.0224 & $2.984 \mathrm{E} 5$ & 0.890 & 0.0388 & 0.4966 & 338.4 & 2.038 & 0.0021 & 6.8731 & 10.82 & 1.993 & 0.0008 \\
\hline 0.0247 & $3.490 \mathrm{E} 5$ & 0.868 & 0.0499 & 0.5488 & 229.5 & 2.029 & 0.0015 & 8.2001 & 434.9 & 1.993 & 0.0423 \\
\hline 0.0275 & $4.093 \mathrm{E} 5$ & 0.833 & 0.0649 & 0.6065 & 187.2 & 2.022 & 0.0014 & 9.3392 & 539.8 & 1.994 & 0.0599 \\
\hline 0.0302 & $5.297 \mathrm{E} 5$ & 0.791 & 0.0921 & 0.6679 & 159.6 & 2.016 & 0.0013 & 10.521 & 357.5 & 1.994 & 0.0447 \\
\hline 0.0334 & 7.601E5 & 0.743 & 0.1463 & 0.7361 & 135.2 & 2.012 & 0.0012 & 11.673 & 130.0 & 1.993 & 0.0180 \\
\hline 0.0364 & $1.002 \mathrm{E} 6$ & 0.716 & 0.2088 & 0.8198 & 110.6 & 2.008 & 0.0011 & 13.478 & 4.401 & 1.993 & 0.0007 \\
\hline 0.0413 & 1.318E6 & 0.691 & 0.3061 & 0.9067 & 100.3 & 2.005 & 0.0011 & 15.567 & 8.455 & 1.993 & 0.0016 \\
\hline 0.0442 & $1.500 \mathrm{E} 6$ & 0.684 & 0.3660 & 1.0063 & 81.14 & 2.002 & 0.0010 & 16.836 & 22.83 & 1.993 & 0.0046 \\
\hline 0.0494 & $1.767 \mathrm{E} 6$ & 0.676 & 0.4642 & 1.1052 & 73.99 & 2.001 & 0.0010 & 18.026 & 81.64 & 1.993 & 0.0175 \\
\hline 0.0561 & $2.226 \mathrm{E} 6$ & 0.661 & 0.6143 & 1.2140 & 67.44 & 1.999 & 0.0010 & 19.834 & 142.5 & 1.994 & 0.0336 \\
\hline 0.0617 & 2.719E6 & 0.688 & 0.7589 & 1.3521 & 60.62 & 1.998 & 0.0010 & 20.666 & 84.96 & 1.994 & 0.0209 \\
\hline 0.0685 & $3.131 \mathrm{E} 6$ & 0.744 & 0.9170 & 1.5006 & 51.88 & 1.997 & 0.0009 & 22.878 & 73.73 & 1.994 & 0.0200 \\
\hline 0.0725 & $3.239 \mathrm{E} 6$ & 0.793 & 1.0123 & 1.6482 & 47.26 & 1.996 & 0.0009 & 25.624 & 93.56 & 1.994 & 0.0285 \\
\hline 0.0826 & 2.981E6 & 0.917 & 1.2159 & 1.8166 & 42.90 & 1.995 & 0.0009 & 28.680 & 68.44 & 1.994 & 0.0233 \\
\hline 0.0946 & 2.099E6 & 1.149 & 1.5429 & 2.0162 & 36.47 & 1.995 & 0.0009 & 32.369 & 44.98 & 1.994 & 0.0173 \\
\hline 0.1062 & $1.215 \mathrm{E} 6$ & 1.564 & 1.7640 & 2.1915 & 33.56 & 1.994 & 0.0009 & 35.291 & 21.32 & 1.994 & 0.0089 \\
\hline 0.1181 & 7.240E5 & 2.080 & 1.8828 & 2.4239 & 26.40 & 1.994 & 0.0008 & 38.699 & 8.142 & 1.994 & 0.0037 \\
\hline 0.1303 & $3.738 \mathrm{E} 5$ & 2.870 & 0.6377 & 2.5208 & 33.03 & 1.994 & 0.0010 & 43.177 & 4.220 & 1.994 & 0.0022 \\
\hline 0.1423 & $1.882 \mathrm{E} 5$ & 3.124 & 0.8535 & 2.7167 & 27.36 & 1.994 & 0.0009 & 46.804 & 2.373 & 1.994 & 0.0013 \\
\hline 0.1592 & 8.657E4 & 2.793 & 0.3182 & 2.7753 & 233.4 & 1.994 & 0.0077 & 53.746 & 1.161 & 1.994 & 0.0007 \\
\hline 0.1807 & $4.226 \mathrm{E} 4$ & 2.520 & 0.1404 & 2.9240 & 638.1 & 1.994 & 0.0222 & 60.340 & 0.983 & 1.994 & 0.0007 \\
\hline 0.2019 & $2.399 \mathrm{E} 4$ & 2.374 & 0.0790 & 3.4233 & 217.2 & 1.994 & 0.0088 & 66.686 & 0.889 & 1.994 & 0.0007 \\
\hline 0.2231 & $1.411 \mathrm{E} 4$ & 2.285 & 0.0477 & 3.6873 & 79.40 & 1.993 & 0.0035 & 73.700 & 0.804 & 1.994 & 0.0007 \\
\hline 0.2466 & 8320 & 2.218 & 0.0294 & 4.1531 & 14.17 & 1.993 & 0.0007 & 81.450 & 0.728 & 1.994 & 0.0007 \\
\hline 0.2747 & 4984 & 2.165 & 0.0187 & 4.4645 & 13.21 & 1.993 & 0.0007 & 90.017 & 0.659 & 1.994 & 0.0007 \\
\hline 0.3012 & 3209 & 2.131 & 0.0129 & 4.7597 & 26.83 & 1.993 & 0.0015 & 98.620 & 0.601 & 1.994 & 0.0007 \\
\hline 0.3329 & 2084 & 2.102 & 0.0090 & 5.2659 & 25.81 & 1.993 & 0.0016 & 109.95 & 0.539 & 1.994 & 0.0007 \\
\hline 0.3679 & 1312 & 2.080 & 0.0062 & 5.6899 & 685.1 & 1.994 & 0.0460 & & & & \\
\hline 0.4066 & 827.2 & 2.063 & 0.0042 & 6.0254 & 279.1 & 1.993 & 0.0200 & & & & \\
\hline
\end{tabular}


extinction curve at the position of the diamond band edge $(6.9 \mathrm{eV})$. Lewis et al. (1989) estimated a detection threshold of this structure of about $10 \%$ of the carbon present as diamonds. This threshold should be increased as well to account for the shallower band edge of the meteoritic nano-diamonds compared to the data used by Lewis et al. (1989).

For the energetic and hydrodynamic modelling of environments possibly containing significant amounts of diamond dust, it will be important to consider the infrared properties of these particles. Our results indicate that their mass absorption coefficient in the visible and IR is about two orders of magnitude lower than that of amorphous carbon particles. That would not have been expected from previous results e.g. by Bernatowicz et al. (1990), which indicated the presence of a thick amorphous-carbon mantle of up to $46 \%$ of the particle's volume. We find that a core-mantle model is probably not well suited for the description of meteoritic nano-diamonds because our spectroscopic results would be consistent only with an unphysical mantle volume fraction of not more than $1 \%$ (see Fig. 7).

\section{Conclusions}

We have performed an extended spectroscopic study of presolar nano-diamonds from the Allende meteorite to close the gaps in the currently known optical data of this material. Different spectroscopic techniques have been used to obtain a consistent complete absorption spectrum of our sample of meteoritic nano-diamonds and to derive the optical constants. Our results indicate that the limits on the amount of diamond grains which can be present in space within the current observational restraints are about a factor of 1.5 higher than previously estimated by Lewis et al. (1989). Furthermore, the mass absorption coefficient at visible and IR wavelengths is about two orders of magnitude lower than that of amorphous carbon, which is not consistent with the presence of a thick amorphous-carbon mantle. These results can be used to improve current models of the environments where nano-diamonds are expected to be present. Further studies on the optical properties of nano-diamonds in a wide spectral range would be valuable, in order to investigate their dependence on surface processing in the lab and in space.

Acknowledgements. The authors would like to thank Gabriele Born for help with the preparational work. Further, we thank R. Schlögl, D. Su, H. Sauer, M. Willinger, and J.-O. Müller of the Fritz-HaberInstitut Berlin for the possibility to perform the EELS measurements and their help. This work has been supported by DFG grant Mu 1164/4 within the DFG Research Unit "Laboratory Astrophysics". It is also part of a joint effort in laboratory astrophysics between the University of Jena and the MPI for Astronomy Heidelberg. A.C.A. acknowledges support from the Carlsberg Foundation.

\section{References}

Alexander, C. M. O’D., Swan, P., \& Walker, R. M. 1990, Nature, 348, 715

Amari, S., Lewis, R. S., \& Anders, E. 1994, Geochim. Cosmochim. Acta, 58, 459

Andersen, A. C., Jørgensen, U. G., Nicolaisen, F., Sørensen, P. G., \& Glejbøl, K. 1998, A\&A, 330, 1080
Allamandola, L. J., Sandford, S. A., \& Tielens, A. G. G. M. 1992, A\&A, 399, 134

Banhart, F., Lyutovich, Y., Braatz, A., et al. 1998, Meteorit. Planet. Sci., 33, A12

Bernatowicz, T. J., Gibbons, P. C., \& Lewis, R. S. 1990, ApJ, 359, 246

Blake, D. F., Freund, F., Krishnan, K. F. M., et al. 1988, Nature, 332, 611

Bohren, C. F., \& Huffman, D. R. 1983, Absorption and Scattering of Light by Small Particles (New York: John Wiley \& Sons)

Braatz, A. 2000, Ph.D. Thesis, Friedrich Schiller University Jena

Braatz, A., Ott, U., Henning, Th., Jäger, C., \& Jeschke, G. 2000, Meteorit. Planet. Sci., 35, 75

Brooke, T. Y., Sellgren, K., \& Smith, R. G. 1996, ApJ, 459, 209

Brooke, T. Y., Sellgren, K., \& Geballe, T. R. 1999, ApJ, 517, 883

Clayton, D. D. 1989, ApJ, 340, 613

Clayton, D. D., Mayer, B. S., Sanderson, C. I., et al. 1995, ApJ, 447, 894

Clayton, G. C., Gordon, K. D., Salama, F., et al. 2003, ApJ, 592, 947

Colangeli, L., Mennella, V., Stephens, J. R., \& Bussoletti, E. 1994, A\&A, 284, 583

Dai, Z. R., Bradley, J. P., Joswiak, D. J., et al. 2002, Nature, 418, 157

Daniels, J., v. Festenberg, C., Raether, H., \& Zeppenfeld, K. 1970, Springer Tracts in Modern Physics, 94, 77

Dorschner, J., Henning, Th., Jäger, C., Mutschke, H., \& Ott, U. 1996, Meteorit. Planet. Sci., 31, A37

Edwards, D. F., \& Philipp, H. R. 1985, in Handbook of Optical Constants of Solids, ed. E. D. Palik (Orlando: Academic Press), 665

Egerton, R. F. 1996, Electron Energy Loss Spectroscopy in the Electron Microscope, 2nd ed. (New York: Plenum Press)

Evans, S. 1992, Surface properties of diamond, in The Properties of Natural and Synthetic Diamond, ed. J. E. Field (London: Academic Press), 181

Evans, S. 1994, Reactivity of diamond surfaces, in Properties and Growth of Diamond, ed. G. Davies (London: INSPEC), EMIS Datareviews Ser., 9, 64

Falke, U., Weber, A., \& Ullmann, J. 1995, Microsc. Microanal. Microstruct., 6, 113

Fitzpatrick, E. L., \& Massa, D. 1986, ApJ, 307, 286

Fraundorf, P., Fraundorf, G., Bernatowitz, T., et al. 1989, Ultramicroscopy, 27, 401

Guillois, O., Ledoux, G., \& Reynaud, C. 1999, ApJ, 521, L133

Hill, H. G. M., d'Hendecourt, L. B., Perron, C., \& Jones, A. P. 1997, Meteor. Planet. Sci., 32, 713

Hill, H. G. M., Jones, A. P., \& d'Hendecourt, L. B. 1998, A\&A, 336, L41

Huss, G. R. 1990, Nature, 347, 159

Huss, G., \& Lewis, R. S. 1995, Geochim. Cosmochim. Acta, 52, 115

Jäger, C. 1997, Ph.D. Thesis, Friedrich Schiller University Jena

Jäger, C., Mutschke, H., \& Henning, Th. 1998, A\&A, 332, 291

Jones, A. P., \& d'Hendecourt, L. 2000, A\&A, 355, 1191

Jones, A. P., d'Hendecourt, L. B., Sheu, S.-Y., et al. 2004a, A\&A, 416, 235

Jones, A. P., d'Hendecourt, L. B., Sheu, S.-Y., et al. 2004b, A\&A, 355, 1191

Jørgensen, U. G. 1988, Nature, 332, 702

Jørgensen, U. G., \& Andersen, A. C. 1999, in The Carbon Star Phenomenon, ed. R. F. Wing (Kluwer), IAU Symp., 177, 349

Koike, C., Wickramasinghe, C., Kano, N., et al. 1995, MNRAS, 277, 986

Kwok, S., Volk, K. M., \& Hrivnak, B. J. 1989, ApJ, 345, L51

Kwok, S., Volk, K. M., \& Hrivnak, B. J. 1999, in Asymptotic Giant Branch Stars, ed. T. Le Bertre, A. Lebre, \& C. Waelkens, IAU Symp., 191, 297 
Lambert, D. L. 1992, A\&AR, 3, 201

Lewis, R. S., Tang, M., Wacker, J. F., et al. 1987, Nature, 326, 160 Lewis, R. S., Anders, E., \& Draine, B. T. 1989, Nature, 339, 117 Lewis, R. S., Huss, G. R., \& Lugmair, G. 1991, Lunar Planet. Sci., 22, 807

Mutschke, H., Dorschner, J., Henning, Th., et al. 1995, ApJ, 454, L157 Ossenkopf, V., Henning, Th., \& Mathis, J. S. 1992, A\&A, 261, 567 Ott, U. 1996, ApJ, 463, 344

Ott, U. 2003, in Astromineralogy (Berlin: Springer), ed. Th. Henning, 236

Papoular, R. 2000, A\&A, 362, L9

Pflüger, J., \& Fink, J. 1991, in Handbook of Optical Constants of Solids II, ed. E. D. Palik (Boston: Academic Press), 293

Posch, Th., Mutschke, H., \& Andersen, A. C., ApJ, submitted

Russell, S. S., Arden, J. W., \& Phillinger, C. T. 1991, Science, 254, 1188
Sandford, S. A. 1996, Meteorit. Planet. Sci., 31, 449

Seki, J., \& Yamamoto, T. 1980, Ap\&SS, 72, 79

Sheu, S.-Y., Lee, I.-P., Lee, Y. T., \& Chang, H.-C. 2002, ApJ, 581, L55

Speck, A. K., \& Hofmeister, A. M. 2003, ApJ, in press

Stecher, T. P. 1965, ApJ, 142, 1683

Tang, M., \& Anders, E. 1988, Geochim. Cosmochim. Acta, 52, 1235

Tielens, A. G. G. M., Seab, C. G., Hollenbach, D. J., \& McKee, C. F. 1987, ApJ, 319, L109

van Kerckhoven, C., Tielens, A. G. G. M., \& Waelkens, C. 2002, A\&A, 384, 568

Virag, A., Zinner, E. K., Lewis, R. S., \& Tang, M. 1989, Lunar Planet. Sci., 20, 1158

Volk, K. M., Kwok, S., \& Hrivnak, B. J. 1999, ApJ, 516, L99

von Helden, G., Tielens, A. G. G. M., van Heijnsbergen, D., et al. 2000, Science, 288, 313

Zinner, E. 1998, Annu. Rev. Earth Planet. Sci., 26, 147 